\title{
Outer Segment Oligomerization of Rds: Evidence from Mouse Models and Subcellular Fractionation ${ }^{\dagger}$
}

\author{
Dibyendu Chakraborty $\ddagger$, Xi-Qin Ding ${ }^{\ddagger}$, Steven J. Fliesler§, and Muna I. Naash ${ }^{*}, \ddagger$ \\ Department of Cell Biology, University of Oklahoma Health Sciences Center, Oklahoma City, \\ Oklahoma 73126, and Departments of Ophthalmology and Pharmacological \& Physiological \\ Science, Saint Louis University School of Medicine, St. Louis, Missouri 63104
}

\section{Abstract}

Retinal degeneration slow (Rds) is a photoreceptor-specific tetraspanin glycoprotein essential for photoreceptor outer segment (OS) morphogenesis. Over 80 mutations in this protein are associated with several different retinal diseases. Rds forms a mixture of disulfide-linked homomeric dimers, octamers, and higher-order oligomers, with Cys 150 playing a crucial role in its oligomerization. Rds also forms noncovalent homo- and hetero-tetramers with its nonglycosylated homologue, Rom-1. Here, we evaluated the subcellular site of Rds oligomerization and the pattern of Rds/Rom- 1 complex assembly in several types of knockout mice, including rhodopsin ( $\mathrm{Rho}^{-/-}$, lacking rod OS), Rom-1 $\left(\right.$ Rom- $\left.1^{-/-}\right)$, neural retina leucine zipper $\left(\mathrm{Nrl}^{-/-}\right.$, cone-dominant), and in comparison with wild-type (WT, rod-dominant) mice. Oligomerization and the pattern of complex assembly were also evaluated in OS-enriched vs OS-depleted preparations from WT and Rom- $1^{-1-}$ retinas. Velocity sedimentation under reducing- and nonreducing conditions and co-immunoprecipitation experiments showed the presence of Rds mainly as homo- and hetero-tetramers with Rom-1 in the photoreceptor inner segment (IS), while higher-order, disulfide-linked intermediate complexes and oligomers were exclusively present in the photoreceptor OS. Rom-1-independent oligomerization of Rds was observed in Rom-1 ${ }^{-/-}$retinas. The pattern of Rds complexes in cones from $\mathrm{Nrl}^{-/-}$mice was comparable to that in rods from WT mice. On the basis of these findings, we propose that Rds traffics from the IS to the OS as homo- and hetero-tetramers, with subsequent disulfide-linked oligomerization occurring concomitant with OS disc morphogenesis (at either the base of OS or the tip of the connecting cilium). These results suggest that Rds mutations that interfere with tetramer formation can block Rds trafficking to the OS, leading to loss-of-function defects.

Vertebrate rod and cone photoreceptors are highly complex neuronal cells that contain the components necessary for the initial events involved in the process of vision. These cells possess a structure called the "outer segment" $\left(\mathrm{OS}^{1}\right)$, a unique organelle composed of hundreds of flattened membranous "discs", the subcellular sites wherein the initial events of phototransduction take place. The inner segment (IS) functions as the cell body of the

\footnotetext{
${ }^{\dagger}$ This study was supported by grants from the National Institutes of Health [EY10609 (M.I.N.), EY16201 (M.I.N.), and EY07361 (S.J.F.)], the Foundation Fighting Blindness (M.I.N.), the Oklahoma Center for the Advancement of Science and Technology (OCAST), Oklahoma City, and the Norman J. Stupp Foundation Charitable Trust (S.J.F.), and by an unrestricted departmental grant from Research to Prevent Blindness (S.J.F.).

*To whom correspondence should be addressed: Muna I. Naash, Ph.D., University of Oklahoma Health Sciences Center, 940 Stanton L. Young Blvd., BMSB 781, Oklahoma City, OK 73126, (405) 271-8001 ext 47969 (voice), (405) 271-3548 (fax), munanaash@ouhsc.edu.

₹University of Oklahoma Health Sciences Center.

$\S_{\text {Saint Louis University School of Medicine. }}$

SUPPORTING INFORMATION AVAILABLE

Sedimentation profile of standard protein markers (Figure S1) and characterization of OS enriched and OS-depleted preparations (Figure $\mathrm{S} 2)$. This material is available free of charge via the Internet at http://pubs.acs.org.
} 
photoreceptor, and is linked to the OS by a connecting cilium. Retinal degeneration slow (Rds) and rod outer segment membrane protein 1 (Rom-1) are members of a tetra-spanning family present along the OS rim membranes $(1,2)$. Rds (also known as peripherin/Rds or peripherin-2) is required for proper photoreceptor OS morphogenesis and stabilization, since retinal degeneration slow $\left(\mathrm{Rds}^{-l-}\right)$ mutant mice fail to develop OSs while heterozygous $\left(\mathrm{Rds}^{+/-}\right)$mice form highly disorganized OS structures consisting of dysmorphic whorls of membranes $(3,4)$. Moreover, Rds gene mutations have been found to associate with a variety of inherited human retinal diseases, including autosomal dominant retinitis pigmentosa (ADRP) and multiple classes of macular degeneration $(5,6)$.

The C-terminus of Rds has been shown to promote membrane fusion in vitro, signifying a possible role in OS renewal (7), while the large intradiscal loop is known to play an important role in protein-protein interactions necessary for disc formation and stabilization (8-11). Rds also has been shown to associate with the rod cyclic nucleotide-gated (CNG) channel through the glutamic acid-rich protein (GARP) of the channel $\beta$ subunit, which is thought to maintain the spatial arrangement of the disc so as to connect the disc rim region to the rod plasma membrane (12).

Our recent work has shown that Rds plays different roles in cone versus rod photoreceptors $(13,14)$. Rod photoreceptors lacking Rds fail to form OS structures; hence, they exhibit minimal phototransduction activity and subsequently degenerate and die. In contrast, cone photoreceptors lacking Rds have markedly altered OS structure, with aberrant lamellar organization but are viable and capable of robust phototransduction (13).

Rds shares many common structural features with its nonglycosylated counterpart, Rom-1 $(2,15)$. Both contain four transmembrane domains with short cytoplasmic $\mathrm{N}$ - and $\mathrm{C}$-terminal regions and a large intradiscal loop of approximately 150 amino acids harboring seven highly conserved cysteine residues $(2,15,16)$. Both in vivo and in vitro studies have demonstrated that noncovalent interactions between Rds and Rom-1 act to support the formation of homo- and hetero-tetrameric functional complexes $(8,17)$. Although the proper assembly of Rds and Rom- 1 complexes is believed to play a crucial role in promoting and preserving normal OS structure, the functional activities and the sites of interaction between the two proteins at the molecular level are not completely understood. It is believed that the hetero-and homotetrameric complexes are linked together through intermolecular disulfide bonds to form octamers and higher-order oligomers that are crucial for disc rim formation (18). This oligomerization has been suggested to be mediated by a cysteine residue at position 150 (Cys150) in the large intradiscal loop $(9,18)$. In contrast to Rds, Rom-1 appears to play a lesser role in disc morphogenesis and structural maintenance, since Rom-1 knockout (Rom- $1^{-/-}$) mice develop OSs whose morphology is only minimally or moderately abnormal (19). Additionally, unlike Rds, Rom-1 is not present in lower vertebrates (20-22), and previous work has demonstrated that Rds is essential for targeting Rom-1 to the OS (23). Furthermore, there is a direct correlation between Rds levels and the structural and functional properties of photoreceptor OSs (24).

The present study was designed to elucidate the intracellular compartment of the photoreceptor cell wherein Rds oligomerization occurs. In particular, we examined more directly the role of

\footnotetext{
${ }^{1}$ Abbreviations: ADRP, autosomal dominant retinitis pigmentosa; CNG, cyclic nucleotide-gated; COS, cone outer segment; CT, Cterminus; DAPI, 4,6-diamidino-2-phenylindole; DTT, dithiothreitol; EM, electron microscopy; FITC, fluorescein isothiocyanate; GARP, glutamic acid rich protein; INL, inner nuclear layer; IP, immunoprecipitation, IS, inner segment; LM, light microscopy; NEM, $N$ ethylmaleimide; Nrl, neural retina leucine zipper; PBS, phosphate-buffered saline; PMSF, phenylmethy-l-sulfonyl fluoride; ONL, outer nuclear layer; OS, outer segment; Rds, retinal degeneration slow; Rho, rhodopsin; Rom-1, rod outer segment membrane protein 1; ROS, rod outer segment; SDS-PAGE, sodium dodecyl sulfate polyacrylamide gel electrophoresis; RPE, retinal pigment epithelium; TBST, Tris-buffered saline containing $0.1 \%$ Tween 20 ; WT, wild-type.
} 
disulfide-mediated oligomerization of Rds in the process of OS disc morphogenesis. Using a variety of well-characterized mouse strains, we show that Rds oligo-merization occurs in the OS of photoreceptors, that Rds can build higher-order complexes with or without Rom-1, and that Rds homo- and hetero-tetramers are formed mainly in the IS of photoreceptors. This is the first in vivo study demonstrating OS oligomerization of Rds and IS trafficking of Rds and Rom-1 core tetrameric complexes.

\section{EXPERIMENTAL PROCEDURES}

\section{Animals}

Retinas from Rom- $1^{-1-}$ mice were utilized for assessing homotypic Rds complex formation in the absence of Rom-1 (19). Rho ${ }^{-1-}$ mice were used to evaluate the pattern of complex formation in retinas lacking rod OS (25). These mice offer a useful model to determine the pattern of Rds and Rom- 1 complex formation in the rod IS and connecting cilium. $\mathrm{Nrl}^{-/-}$mice were used for the study of Rds/Rom-1 complex formation in cone-enriched retinas (26). All experiments and animal maintenance were approved by the local Institutional Animal Care and Use Committee (IACUC; University of Oklahoma Health Sciences Center, Oklahoma City, OK) and conformed to the guidelines on the care and use of animals adopted by the Society for Neuroscience and the Association for Research in Vision and Ophthalmology (Rockville, MD).

\section{Histological and Ultrastructural Analyses}

Methods used for tissue collection and processing for plastic-embedment light and electron microscopy (EM) were as previously described (27). Tissue sections were obtained with a Reichert-Jung Ultracut E microtome using glass or diamond knives. For light microscopy (LM), thick $(0.75-1 \mu \mathrm{m})$ sections were viewed and photographed with an Olympus BH-2 photomicroscope in the auto-expose mode, using $20 \times$ or $60 \times$ DPlanApo objectives, and images were collected with a Nikon digital camera system. For EM, Spurr's resin embedded tissue sections were viewed with a JEOL 100 EX electron microscope.

\section{Immunofluorescence Microscopy}

Eyes were fixed in Davidson's fixative [30 mL of $95 \%(\mathrm{v} / \mathrm{v})$ ethanol, $20 \mathrm{~mL}$ of $10 \%$ neutral buffered formalin, $10 \mathrm{~mL}$ of glacial acetic acid and $30 \mathrm{~mL}$ of distilled water] overnight at $4{ }^{\circ}$ $\mathrm{C}$ (28). The eyes were then washed three times in phosphate-buffered saline (PBS) and stored in $70 \%$ ethanol for subsequent serial dehydration and embedment in paraffin. Eyes were sectioned ( $6 \mu \mathrm{m}$ thickness) on a standard microtome and then annealed onto glass slides at 60 ${ }^{\circ} \mathrm{C}$. Gross retinal morphology was initially assessed by LM following hematoxylin/eosin staining of tissue sections.

Retinal sections were blocked for $1 \mathrm{~h}$ in PBS with 5\% BSA and then incubated overnight at 4 ${ }^{\circ} \mathrm{C}$ with the primary antibodies diluted with PBS in $1 \%$ BSA. Polyclonal antibodies against residues 331-346 of Rds C-terminus (Rds-CT) and residues 336-351 of the Rom-1 C-terminus (Rom-1-CT) were used for detection of Rds and Rom-1, respectively, at a dilution of 1:1000 (10). A polyclonal antibody to opsin (29) was applied at a dilution of 1:1000. All primary antibodies were allowed to incubate with the tissue sections overnight at $4{ }^{\circ} \mathrm{C}$ in a humidified chamber. Sections were then rinsed three times (10 min each) in PBS and incubated with either FITC-conjugated goat anti-rabbit IgG (for sections treated with anti-bovine opsin) or Cy3conjugated goat anti-rabbit IgG (for sections treated with anti-Rds-CT or anti-Rom-1-CT) at a dilution of 1:100 for $1 \mathrm{~h}$ at room temperature in the dark. The sections were then washed three times with PBS, cover slipped with Vectashield containing DAPI stain (Vector Laboratory Inc., Burlingame, CA), and photographed with a Zeiss universal microscope. Digitally captured images were imported into Adobe Photoshop 7.0, where color micrographs were generated and annotated. 


\section{Subcellular Fractionation To Obtain OS-Enriched and OS-Depleted Extracts}

ROS were prepared as previously described (10). Retinas from 4- to 5-week-old mice were dissected and immersed in ice cold homogenization buffer ( $20 \mu$ L/retina) containing $20 \mathrm{mM}$ Tris-acetate, $\mathrm{pH} 7.2,0.25 \mathrm{mM} \mathrm{MgCl}_{2}, 8 \mathrm{mM}$ taurine, $8 \mathrm{mM}$ D-glucose, $20 \%$ (w/v) sucrose, and complete EDTA-free protease inhibitor cocktail (Roche Diagnostics Corporation, Indianapolis, IN). Retinas (10 per preparation) were vortexed at the highest setting (three times, $30 \mathrm{~s}$ each), and supernatant fractions were collected after centrifugation at $3000 \mathrm{~g}$ for $1 \mathrm{~min}$. The three combined supernatants were carefully layered onto a discontinuous sucrose gradient consisting of $0.8 \mathrm{~mL} 40 \%$ (w/v) sucrose and $0.8 \mathrm{~mL} 25 \%$ (w/v) sucrose in homogenization buffer and centrifuged at $61000 \mathrm{~g}$ for $30 \mathrm{~min}$ at $4{ }^{\circ} \mathrm{C}$ in a swinging bucket rotor (Sorvall no. S55S-1009). A pink ROS layer was visualized at the $25 \%-40 \%$ sucrose interface, collected, diluted in homogenization buffer without sucrose at triple the volume, and then centrifuged at $104000 \mathrm{~g}$ for $30 \mathrm{~min}$ at $4{ }^{\circ} \mathrm{C}$ to obtain the ROS pellet in a fixed angle rotor (Sorval no. S150AT-0148). An OS-depleted fraction was generated from the remaining portion of the retinas. The ROS pellet was solubilized using buffer containing $2 \times$-strength PBS, $\mathrm{pH} 7.5,2 \%$ Triton X-100, $5 \mathrm{mM}$ NEM. After $20 \mathrm{~min}$ incubation on ice, extracts were centrifuged at 90 $000 \mathrm{~g}$ for $30 \mathrm{~min}$ at $4{ }^{\circ} \mathrm{C}$ in a Sorvall Discovery M150 centrifuge (Sorvall no. S150AT-0148) to remove remaining particulates, and the supernatants were collected for further analysis.

\section{Western Blot and Immunoprecipitation Analysis of Rds and Rom-1}

Rds-CT and Rom-1-CT polyclonal antibodies were used for Western blot and immunoprecipitation as described previously (10). Dissected retinas were homogenized on ice in solubilization buffer containing $50 \mathrm{mM}$ Tris- $\mathrm{HCl}, \mathrm{pH} 7.5,100 \mathrm{mM} \mathrm{NaCl}, 5 \mathrm{mM}$ EDTA, $1 \%$ Triton X-100, 0.05\% SDS, $2.5 \%$ glycerol, and $1.0 \mathrm{mM}$ PMSF. After solubilization at $4{ }^{\circ} \mathrm{C}$ for $1 \mathrm{~h}$, the homogenates were centrifuged at $104000 \mathrm{~g}$ for $30 \mathrm{~min}$, and the supernatants were measured by the Bio-Rad Protein Assay. For Western blotting, retinal extracts (each containing $10 \mu \mathrm{g}$ of protein) were incubated for at least $30 \mathrm{~min}$ at room temperature with Laemmli sample buffer [50 mM Tris-HCl, 2\% SDS, $100 \mathrm{mM}$ dithiothreitol (DTT), and 10\% glycerol], separated by $10 \%$ SDS-PAGE, and transferred onto a PVDF membrane in transfer buffer containing 25 $\mathrm{mM}$ Tris-base, $190 \mathrm{mM}$ glycine, $20 \%$ methanol, and $0.03 \%$ SDS. The blots were blocked with $5 \%$ nonfat dry milk in TBST (Tris-buffered saline containing $0.1 \%$ Tween 20 ) at $4{ }^{\circ} \mathrm{Covernight}$ before incubation with primary antibodies. Anti-Rds-CT, anti-Rom-1-CT, and anti-1D4 (a monoclonal antibody to opsin; generously provided by Dr. Robert Molday, University of British Columbia, Canada) each were used at a dilution of 1:1000, while the monoclonal antiactin antibody (ABCAM, Cambridge, MA) was used at a dilution of 1:250. The primary antibodies were allowed to incubate with the PVDF membrane for $2 \mathrm{~h}$ at room temperature and then washed three times with TBST (15 min each). PVDF membranes were then incubated with either goat-anti-mouse IgG at a dilution of 1:25 000 (for blots exposed to anti-1D4 or actin antibodies) or goat-anti-rabbit antibody at a dilution of 1:25000 (for blots exposed to Rds-CT or Rom-1-CT antibodies) for $1 \mathrm{~h}$ at room temperature. After four 15-min washes with TBST, the blots were exposed to film after using SuperSignal chemiluminescent substrate (Pierce, Rockford, IL). The films were scanned in the scanner (Multilmage Light Cabinet, Alpha Infotech Corporation) and after proper calibration. AlphaEaseFc software was used to measure the intensity of each band. Taking the sum of all band intensities as $100 \%$, the intensity level of each band was presented as percentage of the total for that blot.

For immunoprecipitation, $100 \mu \mathrm{g}$ of retinal extracts or $100 \mu \mathrm{L}$ of sucrose gradient fractions were incubated with either anti-Rds antibody or anti-Rom-1 antibody (at 1:100 dilution) for at least $2 \mathrm{~h}$ at $4{ }^{\circ} \mathrm{C}$. A suspension of protein A-Sepharose beads ( $25 \mu \mathrm{L}$; Sigma-Aldrich Corp, St. Louis, MO) was added to each sample and allowed to incubate at $4{ }^{\circ} \mathrm{C}$ overnight, in solubilization buffer. After adsorption, the beads were washed three times with solubilization 
buffer and bound proteins were eluted with Laemmli sample buffer for immunoblotting analysis using the respective antibodies as described previously.

\section{Velocity Sedimentation Analysis}

Reducing and nonreducing velocity sedimentations were performed using a previously established procedure on whole retinal extracts (10). In brief, continuous density gradients of $5-20 \%$ sucrose were prepared by sequentially layering $0.5 \mathrm{~mL}$ each of PBS-buffered 20,15 , 10 and $5 \%$ sucrose, containing $0.1 \%$ Triton X-100 and $2 \mathrm{mM} \mathrm{DTT}$, in a centrifuge tube for sedimentation analysis of Rds and Rom-1 under reducing conditions. Parallel procedures under nonreducing conditions were performed by replacing the DTT in both the extraction and gradient buffers with $10 \mathrm{mM}$ NEM (N-ethylmaleimide). The gradients were allowed to stabilize at room temperature for $1 \mathrm{~h}$, and then chilled for at least $30 \mathrm{~min}$ on ice prior to loading the samples. Samples ( $200 \mu \mathrm{L}$ each) of either whole retinal, OS-enriched or OS-depleted extracts were gently layered onto the gradients and centrifuged at $109000 \mathrm{~g}(40000 \mathrm{rpm})$ for $16 \mathrm{~h}$ in a Sorvall Discovery M150 centrifuge using swing bucket rotor [Sorvall no. S55S-1009, with initial sample radius $\left(r_{\min }\right)$ of $45.4 \mathrm{~mm}$ ] at $4{ }^{\circ} \mathrm{C}$. The bottom of the centrifuge tube was punctured with a 261/2-gauge needle and gradients were fractionated dropwise into collection tubes (6 drops/tube). Equal volumes of each fraction $(15 \mu \mathrm{L})$ were separated on $10 \%$ SDSPAGE and then processed for Western blotting with Rds-CT and Rom-CT polyclonal antibodies (10). The sizes of Rds and Rom-1 subunit assembly were determined as described before $(10,30)$ and confirmed with several standard protein markers (apoferritin, $M_{\mathrm{r}} \sim 443 \mathrm{kDa}$; beta amylase, $M_{\mathrm{r}} \sim 200 \mathrm{kDa}$; alcohol dehydrogenase, $M_{\mathrm{r}} \sim 150 \mathrm{kDa}$; bovine serum albumin, $M_{\mathrm{r}} \sim 66 \mathrm{kDa}$; and carbonic anhydrase, $M_{\mathrm{r}} \sim 29 \mathrm{kDa}$; Sigma) sedimented at the same time and under similar conditions (see Supporting Information Figure 1). For each experimental paradigm, at least three independent preparations underwent velocity sedimentation and Western blotting as described above. The amount of Rds or Rom-1 in each fraction was calculated as a percent of total immunoreactivity. The levels are presented graphically as mean \pm standard deviation values from all iterations of the given experiment with a representative blot shown in each case. The sedimentation coefficient $\left(S_{20, w}\right)$ was calculated using a well established computer program (QBasic) and as described before (31) where partial specific volume $(v)$ is taken as $0.83 \mathrm{~mL} / \mathrm{g}(9,30)$.

\section{RESULTS}

\section{Retinal Morphology}

We employed several mouse models to evaluate potential differences in the pattern of Rds/ Rom-1 complex formation in the IS and their ultimate site of integration and function in the photoreceptor OS compartment. These models include the following: (a) $\mathrm{Rho}^{-/-}$, lacking OS (IS/connecting cilium-enriched); (b) Rom- $1^{-/-}$(to allow examination of Rds complex formation in the absence of Rom-1); (c) $\mathrm{Nr}^{-/-}$(a rod-free, S-cone-only retina); (d) $\mathrm{Rds}^{-/-}$(a negative control for the absence of Rds and the presence of low amounts of Rom-1); (e) WT, representing the normal rod-dominant, duplex retina (95-97\% rods and 3-5\% cones). Figure $1 \mathrm{~A}$ shows representative micrographs obtained by histological and ultrastructural analyses of retinas from 1-month old mice. As expected, LM (upper panels) and EM (lower panels) images demonstrate typical, well-organized OSs in the WT retina and the lack of OSs in $\mathrm{Rds}^{-/-}$and $\mathrm{Rho}^{-1-}$ retinas $(10,25) . \mathrm{Nrl}^{-1-}$ retinas show cone-like OS structures in the photoreceptor layer and rosette-like structures in the outer nuclear layer (ONL) (13) (26). Nrl is a transcription factor that is a key regulatory molecule required by retinal progenitor cells during photoreceptor development (26). When these progenitor cells fail to receive this cue, they divert

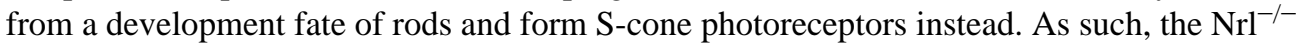
mouse presents an ideal model for studying cones in the absence of rods. Furthermore, 
ultrastructural analysis of the $\mathrm{Nrl}^{-/-}$retina shows considerably shorter OS than in the WT retina (Figure 1A, lower panel) (13).

\section{Expression Levels and Localization of Rds and Rom-1}

To examine the expression levels and cellular localization of Rds and Rom-1 in 1-month old retinas from the different models, we performed immunohistochemistry (Figure 1B) and Western blot analysis (Figure 1C) using antibodies to Rds, Rom-1, and rod-opsin. All three antibodies showed strong and selective immunoreactivity in the OS structures of the WT retina (Figure 1B). As expected, no anti-Rds or anti-Rom-1 immunoreactivity was present in $\mathrm{Rds}^{-/-}$retinas; however, anti-rod-opsin immunoreactivity was found in photoreceptor IS, particularly at the tip of the connecting cilium and in the perinuclear region. $\mathrm{Rho}^{-1-}$ retinas, on the other hand, showed labeling with Rds- and Rom-1-specific antibodies at the tip of the connecting cilium. Rom- $1^{-1-}$ retinas lacked anti-Rom-1 labeling, as expected, but anti-Rds and anti-rod opsin antibodies showed robust staining in OS structures. No rod opsin immunolabeling was detected in the $\mathrm{Nrl}^{-/-}$retinas, whereas strong labeling with anti-Rds and anti-Rom-1 were observed in the OS layer between the retinal pigment epithelium (RPE) and the ONL, along with OS structures inside the rosettes.

To evaluate the level of Rds, Rom-1 and rod opsin in 1-month old retinas from the various mouse strains, we conducted semiquantitative Western blot analysis. Using the rod opsindirected monoclonal antibody, Rho 1D4, ample amounts of rod opsin were detected in retinal extracts from WT and Rom- ${ }^{-/-}$, whereas none were found in the $\mathrm{Rho}^{-/-}$and $\mathrm{Nrl}^{-/-}$extracts (Figure 1C). Rds was present at comparable levels to WT in all mouse strains except in $\mathrm{Rds}^{-/-}$retinas where it was completely absent, and in $\mathrm{Nrl}^{-/-}$retinas, where it was decreased slightly (13). A fair amount of Rom-1 was also detected in WT, Rho ${ }^{-1}$, and, to a lesser level, in $\mathrm{Nrl}^{-/-}$retinal extracts, while a negligible amount was found in the $\mathrm{Rds}^{-1-}$ and none in the Rom- $1^{-l-}$ extracts. In comparison to Rds, Rom- 1 levels were down-regulated in $\mathrm{Nrl}^{-1-}$ retinas (Figure 1C, lane 5). These findings are consistent with those reported previously $(24,26)$. The significant reduction of rod opsin level in the $\mathrm{Rds}^{-/-}$retina reflects the degeneration and loss of photoreceptors and/or the absence of OSs due to the lack of Rds.

\section{Subunit Assembly of Rds and Rom-1 in the Rod-Dominant WT Retina}

To gain insight into the physical properties and composition of Rds and Rom-1 complexes, we subjected retinal extracts from the mouse strains to sucrose density velocity sedimentation analysis. This approach was applied first on WT retinal extracts, either by sedimentation on a reducing gradient followed by reducing SDS-PAGE or by sedimentation on a nonreducing gradient and subsequent nonreducing SDS-PAGE (Figure 2). Twelve fractions were collected from a 5-20\% sucrose gradient and then subjected to Western blot analysis, probing with antiRds and anti-Rom-1 antibodies. Under reducing conditions, Rds complexes were completely dissociated from the core complexes of Rds homo- and hetero-tetramers with Rom-1, which appeared as the main peak centered at fraction 7 for both proteins (Figure 2, panels A, B and E, F) with sedimentation coefficient $S_{20, w}=5.83 \mathrm{~S}$. Similar analysis were performed on the fractions from the nonreducing gradient (Figure 2, panels C, D and G, H). Higher-order homooligomeric complexes of Rds were detected mainly in fractions $1-4$, sedimented with coefficient $S_{20, \mathrm{w}} \sim 12.19 \mathrm{~S}$ and largely consisted of disulfide-linked Rds homo-dimers and, to a lesser extent, of other higher complexes (Figure 2C,D). In contrast, Rom-1 did not participate in these complexes (Figure 2, panels G,H, fractions 1-4), suggesting that these higher-order oligomers detected by the Rds antibody are comprised solely of Rds. Furthermore all Rds in fractions 1-5 from the nonreducing gradients are engaged in disulfide-linked complexes.

Fractions 5-9 from the same gradients (as shown in Figures 2C,D and G,H) sedimented at the same rate as the core tetrameric complexes $\left(\mathrm{S}_{20, \mathrm{w}}=5.83 \mathrm{~S}\right)$ evident under reducing conditions (see gradient profiles, Figure 2A,B and E,F). It is worth noting that the majority of Rds and 
Rom-1 in these fractions were present in a noncovalently linked form. Disulfide-linked form of Rds was detected in fractions 5-9 while disulfide-linked form of Rom-1 was identified in fractions 5-6.

Furthermore, we calculated the percentage of Rds and Rom-1 present in non-disulfide vs disulfide-linked complexes from three independent nonreducing sedimentation and nonreducing Western blot analyses (Figure 2I,J). It is important to note that over $80 \%$ of Rds is present as disulfide-linked complexes, while only $\sim 20 \%$ of it is present in noncovalently linked form. The majority of the disulfide-linked portion of Rds appeared as dimers, with a smaller portion as higher-order complexes. However, over $85 \%$ of Rom-1 is present in noncovalently linked form, while $\sim 15 \%$ exists as disulfide-linked complexes.

\section{Subunit Assembly of Rds and Rom-1 in Retinas from Knockout Mice}

Rds and Rom-1 complex assembly was evaluated in whole retinal extracts prepared from $\mathrm{Rho}^{--}, \mathrm{Nrl}^{--}$, $\mathrm{Rom}-1^{---}$mice, and in comparison to WT controls. In these nonreducing sedimentation experiments, 12 fractions were collected and separated on SDS-PAGE under reducing conditions. $\mathrm{Rho}^{-1-}$ mice were used in this study to evaluate the pattern of Rds complexes in retinas where the rods lack an ROS, but contain an IS and connecting cilium. Interestingly, the sedimentation profiles of Rds and Rom-1 proteins from $\mathrm{Rho}^{-/-}$extracts were not significantly different from those obtained with the WT extracts. However, Rds homooligomeric complexes were much less abundant in the $\mathrm{Rho}^{-1-}$ retina (Figure 3B), as compared to the WT (Figure 3A). It seems that the majority of Rds and Rom-1 are engaged in tetrameric complexes, confirming the results of a previous study that suggests the presence of a checkpoint at or near the connecting cilium that only allows the passage of tetrameric forms of Rds and Rom-1 from the IS to the OS (23).

Extracts from $\mathrm{Nrl}^{-/-}$retinas (Figure 3C) also showed a similar pattern of Rds and Rom-1 sedimentation to that of WT retinas (Figure 3A) but with significantly less involvement of Rom-1, likely due to the lower levels of Rom- 1 in cones. Rom- 1 is present in fractions 4-8 of the WT gradient and in fractions 6-8 of the $\mathrm{Nrl}^{-/-}$gradient. However, the level of higher-order homo-Rds complexes in the $\mathrm{Nrl}^{-1-}$ retinas is similar to that of the WT, suggesting a comparable need for these complexes to build the rod and cone OS rims. Interestingly, the pattern of Rds complex formation in the Rom- $1^{-/-}$retina is also comparable to that seen with WT. Rds was detected in all fractions, indicating the presence of all forms of the complexes (Figure 3D). Regardless of the mouse strain examined, Rom-1 peaks in fraction 7 of the gradients, most likely where hetero-tetrameric complexes tend to fractionate.

\section{Association of Rds and Rom-1 in Retinas from $\mathrm{Rho}^{-/-}$and $\mathrm{Nr}^{-/-}$mice}

Rds and Rom-1 are known to associate with each others to form tetramers, octamers, and higher-order oligomers (18). However, it is not known in what specific subcellular compartment of the photoreceptor cell such complex formation occurs. To test whether such associations take place in the IS vs the OS, we performed co-immuno-precipitation experiments with whole retinal extracts from $\mathrm{Rho}^{-/-}$(OS-free) and in cone-enriched $\mathrm{Nrl}^{-/-}$retinas, using anti-Rds antibodies (Figure 4A). A comparable amount of Rom-1 was immunoprecipitated along with Rds from WT, $\mathrm{Rho}^{-/-}$and $\mathrm{Nrl}^{-/-}$extracts. As expected for the negative control, no Rom-1 was detected in Rom- $1^{-1-}$ extracts. Similar to the WT retina, Rds and Rom-1 were capable of association in the $\mathrm{Rho}^{-1-}$ and $\mathrm{Nrl}^{-/-}$retinas (see Figure 4A, lanes 1, 3, and 5). Since Rom-1 is mainly present in tetrameric assemblies, we performed co-immunoprecipitation analysis on the Rom-1-containing fractions 4-9 from nonreducing gradients of WT and $\mathrm{Rho}^{-/-}$retinal extracts to verify the presence of homo- and/or hetero-tetramers of Rds and Rom-1 (Figure 4B). As shown for WT retinas (Figure 4B, upper two panels), immunoprecipitation with anti-Rds antibodies coprecipitated Rom- 1 mainly from fractions 5 
and 6, while only extremely small amounts were coprecipitated from fractions 4 and 7. It is important to note that the goal of presenting the data in Figure 4 is to demonstrate the existence of physical interactions between Rds and Rom-1 in all fractions containing Rom-1. Since we encountered some technical difficulties with the efficiency of the immunoprecipitation directly from these fractions (likely due to the high sucrose concentrations), it is difficult to make definitive conclusions regarding the structural composition of these complexes. Nevertheless, these data suggest that fraction 7 contains Rds homo-tetramers, while fractions 5 and 6 contain Rds/Rom-1 hetero-tetramers. Interestingly, more hetero-tetramers of Rds and Rom-1 were detected in extracts from $\mathrm{Rho}^{-1-}$ retinas, and were distributed among fractions 4 to 6 . Since all Rom-1 tetramers were found in association with Rds in $\mathrm{Rho}^{-/-}$(OS-free) extracts, we conclude that hetero-tetrameric association occurs in the IS compartment and is the form essential for trafficking Rom-1 to the OS (Figure 4B, lower two panels).

\section{Pattern of Rds and Rom-1 Complexes in WT OS-Enriched and OS-Depleted Preparations}

To evaluate any differences in the pattern of Rds and Rom-1 complex assembly between photoreceptor OS and IS compartments, we performed subcellular fractionation of WT and Rom- $1^{-1-}$ retinas and generated OS-enriched and OS-depleted extracts. The extracts were separated on SDS-PAGE and subjected to Western blot analysis, using OS- and IS-specific probes, to validate the fractionation procedure and the qualitative levels of cross-contamination between the two compartments. Using anti-rhodopsin antibodies and anti GARP-4B1, trace amounts of rod opsin (see Supporting Information for Figure 2, lane 3, left upper panel) and GARP containing proteins (see Supporting Information for Figure 2, lane 3, right upper panel) were detected in the OS-depleted extracts, most likely representing a newly synthesized pool of proteins, while most of the rod opsin and GARP proteins was found in the OS-enriched extracts (see Supporting Information for Figure 2, lane 2, left upper panel and right upper panel, respectively). The $\alpha 3$ isoform of $\mathrm{Na}^{+}-\mathrm{K}^{+}$-ATPase, an IS marker protein, was mainly found in the OS-depleted extract (see Supporting Information for Figure 2, lane 3, lower panel); a small amount was detected in whole retinal extract (lane 1), while none was detected in the OSenriched extract (lane 2), in good agreement with prior studies (32). Immunoreactivity to endoplasmic reticulum (ER) marker, calreticulin, was mainly present in the OS-depleted and whole retinal extracts and none in the OS-enriched extracts. At least four independent preparations were made for the OS-enriched and OS-depleted extracts and each was evaluated for contaminants from the other compartment before it was used in the sedimentation experiment.

Next, we carried out velocity sedimentation and Western blot analysis under reducing (Figure 5A) and nonreducing (Figure 5B) conditions on WT OS-enriched and OS-depleted preparations. By applying a reducing gradient to these samples, we were able to resolve Rds and Rom-1 complexes to their minimum sizes in the absence of disulfide bonding. In the presence of DTT, higher-order complexes of Rds and Rom-1 were reduced to tetramers that appeared as a single peak spanning fractions 7 to 9 from OS-enriched and OS-depleted samples, and fractions 5 to 8 from a Rho ${ }^{-/-}$retinal extract (OS-depleted) (Figure 5A). A similar pattern of sedimentation was seen with all four different OS-enriched/OS-depleted preparations. In contrast, sedimentation analysis under nonreducing gradient conditions revealed the presence of higher-order complexes of Rds mainly in the OS-enriched preparations, predominantly in two main peaks spanning fractions 1 to 8 (Figure 5B, left panels). Furthermore, sedimentation of the OS-depleted samples under nonreducing conditions showed mainly the presence of tetrameric forms of Rds complexes and a small amount of Rds homo-oligomers (Figure 5B, right panels). These data indicate the absence of higher-order Rds complex formation in the OS-depleted preparations; hence, Rds oligomerization occurs in the OS compartment. A difference in the distribution of Rom-1 was also detected between the OS-enriched and OSdepleted preparations. Although one main peak of Rom-1 was detected from both preparations, 
it spanned fractions 1 to 9 of the OS-enriched preparation gradients, while it encompassed fractions 6 to 9 on gradients of the OS-depleted preparations. These data confirm and extend the results obtained from different mouse strains ( $c f$. Figures 2 and 3), as described above, demonstrating that Rom-1 primarily forms hetero-tetrameric complexes with Rds both in the IS and OS, and that higher-order hetero-oligomers are much less prevalent and found only in the OS.

\section{Rds Complex Assembly in Rom-1 ${ }^{-/-}$OS-Enriched and OS-Depleted Preparations}

It has been shown that Rom- $1^{-1-}$ mice are capable of forming functional OS structures (19); however, the extent of Rds complex formation in these retinas has not been investigated. OSenriched and OS-depleted preparations were generated from Rom- ${ }^{-1-}$ retinal extracts and tested for cross-compartmental contamination as indicated above (data not shown). Samples exhibiting negligible cross-contamination were subjected to nonreducing velocity sedimentation followed by reducing SDS-PAGE and Western blot analysis. Figure 6 shows the pattern of Rds distribution from OS-enriched and OS-depleted preparations. Similar to what we observed with WT retinas, the majority of Rds in OS-enriched preparations from Rom- $1^{-1-}$ retinas was present as homo-octamers and higher-order oligomers (Figure 6, fractions 1-6). Interestingly, there was a complete lack of Rds oligomer formation in the OSdepleted samples from Rom- $1^{-1-}$ retinas (Figure 6, lower panel); mainly Rds homo-tetramers were detected. Again, this finding suggests that Rds homo-oligomers are essential for building the OS rim structure, whereas Rds tetramers represent the dominant form in which Rds is trafficked from the IS to the OS. Hence, this presents yet another example to confirm that Rds higher oligomerization occurs in the OS compartment.

\section{DISCUSSION}

In the present study, we examined the intracellular sites and patterns of Rds and Rom-1 complex assembly and oligomerization in retinas from different genetically modified knockout mice and compared with normal (WT, rod-dominant) mice. These knockouts are $\mathrm{Rho}^{-/-}$(an OSdepleted model), Rom- $1^{-/-}$(containing Rds, but no Rom-1), and $\mathrm{Nrl}^{-/-}$(a rod-free, S-conedominant model). Oligomerization and pattern of complex assembly were also evaluated in OS-enriched and OS-depleted preparations from WT and Rom- $1^{-/-}$retinas. Using reducingand nonreducing velocity sedimentation analysis and immunoprecipitation, we found that Rds is mainly present as homo- and hetero-tetramers with Rom-1 in the photoreceptor IS, while higher-order, disulfide-linked complexes (e.g., Rds/Rom-1 hetero-octamers and Rds homooligomers) are mainly present in the photoreceptor OS (Figure 7). We also showed that Rds oligomerization is independent of Rom- 1 , since this occurs even in Rom- $1^{-1-}$ retinas.

Furthermore, no significant differences in the pattern of Rds complexes were detected between rod-dominant WT and S-cone-dominant $\mathrm{Nrl}^{-/-}$retinas despite the structural differences in their OSs. We also found that the majority of Rds is engaged in disulfide-linked complexes, while only a small portion of it is present as noncovalently linked complexes. In contrast, Rom-1 is mainly present in noncovalently linked form, with only a small portion existing in disulfidelinked complexes. Furthermore, our data suggest that Rds traffics from the IS to the OS compartment of the photoreceptor cell as homo- and hetero-tetramers, whereas disulfide-linked oligomerization of Rds occurs in the OS, likely during disc rim formation at the site of disc morphogenesis.

These fundamental new observations provide a basis for expanding current concepts regarding the biological role of Rds/Rom-1 complex formation, and how these might differ in cones vs rods (the latter afforded by our comparison of the rod-dominant WT vs the S-cone-dominant $\mathrm{Nrl}^{-/-}$retina). In addition, the results obtained using OS-enriched vs OS-depleted preparations combined with biochemical data obtained with Rom- $-^{-/-}$retinas allow us to formulate rational 
hypotheses about the possible distinct functions of Rds and Rom-1 in photoreceptor cells. Finally, our results provide new information regarding the regulation of Rds/Rom-1 complex formation and its role in the trafficking of Rds and Rom-1 to OS. These issues are discussed below.

\section{Rds/Rom-1 Complexes in Cones}

We examined the pattern of Rds complex formation in the cone-dominant retina of the $\mathrm{Nrl}^{-/-}$mouse as compared to the WT. We wanted to verify whether any differences in Rds/ Rom-1 complex assembly correlated with the distinct OS structures found in rods (ROS) vs cones (COS). Surprisingly, we detected no significant differences between these two retinas in terms of their pattern of Rds complex formation, based upon nonreducing velocity sedimentation analysis. Thus, differences in the pattern of Rds/Rom- 1 complex assembly cannot account for the ultrastructural differences between the "closed discs" found in ROS vs the "open discs" (or, more correctly, lamellae) present in COS. On the basis of these observations, combined with our previously published studies indicating that Rds does function differently in rods vs cones (13), we hypothesize that the presence of as yet unidentified Rdsinteracting partners may account, in part, for the observed differences in OS morphogenesis between rods and cones.

\section{The Role of Rds/Rom-1 Complexes in Photoreceptors}

Another interesting point raised by our data concerns the biological role of Rom- 1 in photoreceptor cells. Compared to Rds, relatively little is known about the role of Rom-1 in molecular complex formation and trafficking in photoreceptors. The results of recent studies by Papermaster and colleagues (33) using transgenic Xenopus laevis suggest that the Cterminus of Rds is capable of directing Rds/Rom-1 trafficking to the OS. Rom-1, on the other hand, does not have such functionality. This conclusion is supported by indirect evidence we have obtained using $\mathrm{Rds}^{-1-}$ mice, where little or no Rom-1 was detected and no OS morphogenesis occurred $(10,24)$, as well as from results obtained using Rom- $1^{-/-}$mice, where Rds assembles into complexes and is trafficked normally to the OS. A second hypothesized role for Rds/Rom-1 complexes is to mediate membrane fusion requisite for OS rim formation and membrane morphogenesis. Boesze-Battaglia and co-workers have demonstrated that Rds complexes are capable of mediating membrane fusion in vitro (34). More recently, it has been shown that Rom- 1 is not capable of mediating membrane fusion alone but its presence in Rds/ Rom- 1 complexes enhances Rds-mediated membrane fusion (35). The third known function of Rds in the OS is one of the focuses of this work. Here, we have extensively characterized the presence of Rom- 1 in all types of Rds complexes and have shown that the higher-order Rds oligomers (which are thought to be responsible for maintenance of OS rim architecture) contain almost no Rom-1. Rom-1 is present either as an intermediate-size oligomer (octamer) or heterotetramer even in the OS. Also, in good agreement with prior findings by Molday and workers (18), we observed no differences in higher-order complex formation in the OS in the absence of Rom-1 (i.e., Rom- $1^{-/-}$compared with WT).

We propose that a primary role of Rom-1 is to facilitate and regulate Rds-mediated OS membrane fusion. This speculation is consistent with the wider disc spacing observed in the $\mathrm{Rom}^{-1-}$ retina, compared to the WT retina (19). However, the main role of Rds/Rom-1 tetramers in the OS remains to be elucidated. This is important, since studies by us $(10,24)$ and others (18) support the role of higher-order Rds homo-oligomers in building and maintaining OS membrane rim structure. If the structure of the OS rim is maintained by higher-order Rds homo-oligomers, then this begs the question of what function(s) can be ascribed to the stable Rds/Rom-1 hetero-tetramers in the OS? This remains to be answered and will require further investigations to elucidate completely. 


\section{Cellular Distribution of Rds/Rom-1 Complexes}

Previous sedimentation experiments have shown the presence of different ranks of Rds homoand hetero-molecular complexes in the bovine retina and in a heterologous expression system (17). However, information regarding the cellular distribution of these complexes in the photoreceptor cells was heretofore not well-described. It also has not been known where in the photoreceptor cell Rds oligomerization occurs. Nonreducing velocity sedimentation followed by nonreducing Western blot analysis of WT retinal extracts revealed the pattern of Rds and Rom- 1 complex formation and showed the presence of noncovalently linked tetramers and disulfide-linked intermediate and higher-order complexes. Unlike Rom-1, the majority of Rds was engaged in disulfide-linked complexes, with only a small portion present as noncovalently linked tetramers. Rom-1, on the other hand, was found to exist primarily as noncovalently linked tetramers, while only a small portion was involved in disulfide-linked complexes. Again, this further supports the role of Rds in building the OS rim structure.

Velocity sedimentation analysis of retinal extracts under nonreducing conditions, using the various mouse strains employed in the present study, has now revealed the presence of intermediate and higher-order Rds complexes only in the OS compartment, with little or none in the IS compartment. Furthermore, the distinct cellular distribution pattern of Rds oligomers, as compared to their core (tetramers), implies that assembly of these higher-order complexes takes place in the OS, likely in the region of nascent disc membrane assembly or possibly at the tip of the connecting cilium. The significance of these higher-order complexes in building and maintaining the disc rim structure and OS morphology is now well accepted (18). It has been proposed that the connecting cilium serves as a checkpoint for properly formed Rds/ Rom-1 tetramers and suggested that dimers or improperly assembled tetramers will not traffic to the OS (23). Our current work further supports this observation. The idea that higher-order oligomers are formed in the OS is further supported by results obtained using $\mathrm{Rho}^{-/-}$mice.

Although the $\mathrm{Rho}^{-1-}$ retina lacks OS structures, protein levels of Rds and Rom-1 were measured at near WT levels. Both proteins localized to the tip region of the connecting cilium where OS morphogenesis occurs. The results obtained by nonreducing velocity sedimentation analysis as reported here indicate that, in the $\mathrm{Rho}^{-1-}$ retina, the majority of Rds/Rom-1 is present in tetrameric form, either as homo- or hetero-tetramers, whereas higher-order complexes represent only a very small fraction of the total (Figure 3B). We believe that there are two reasons for this. First, the data we show are from P30 $\mathrm{Rho}^{--}$retinas, which still have healthy cones with intact OSs that may contribute to the presence of the low amount of higher-order Rds complexes. This is further supported by the sedimentation analysis results presented in Figure 3C, showing the presence of these higher-order Rds oligomers in cone-dominant $\mathrm{Nrl}^{-1-}$ mouse retinas. Second, in $\mathrm{Rho}^{-1-}$ retinas, Rds higher-order oligomers are probably formed in the distal, periciliary IS zone of rods where initiation of OS morphogenesis is abortively attempted. Recent ultrastructural analysis of this region showed that small membranous sacs emerge from the distal connecting cilium of the 1-month old $\mathrm{Rho}^{-/-}$retinas that are immunoreactive to Rds antibody (36). Therefore, we think that these membranous structures at the tips of $\mathrm{Rho}^{-1-}$ rod connecting cilia are probably nascent disc membrane precursors containing higher-order complexes of Rds that normally would support disc morphogenesis if rod opsin was available. Direct evidence of this would be extremely difficult to demonstrate.

\section{Disulfide Bond-Mediated Rds/Rom-1 Complex Formation}

Intermolecular disulfide bonds play an important role in the assembly of multi-subunit proteins and higher-order oligomeric complexes. Our analysis suggests the presence of two distinct classes of Rds-containing complexes in photoreceptor cells, as detected by velocity sedimentation under nonre-ducing conditions. The first class is composed of nonco-valently associated "core" Rds-homo- and Rds/Rom-1-hetero-tetrameric complexes. Such core 
tetramers have been reported by others (23), and here we have demonstrated their presence in both the OS and IS compartments. The second class consists of disulfide-linked, higher-order Rds homo-oligomers present mainly in the OS (Figure 5B). Treatment of ROS membranes with DTT results in the breakdown of these homo-oligomers into core tetramers (Figure 5A), indicating that the intermolecular disulfide bonds are readily accessible to this reducing agent and are essential for oligomerization. It has been proposed that the association of Rds with Rom-1 in core complexes limits the size of their oligomers and the number of subunits that participate in the complex assembly through intermolecular disulfide bonds. Our studies reveal that both of Rds and Rom-1 form core tetrameric complexes in the IS of the photoreceptor and subsequently form higher-order complexes in the OS. Our studies also demonstrated that Rds and Rom-1 do not form higher-order complexes with equal facility. In fact, we did not observe any involvement of Rom-1 in oligomeric forms of Rds (see Figure 2I,J).

On the basis of the results of our current studies and others, we propose a model wherein Rds homo- and hetero-tetrameric core complexes are likely prevented from higher-order disulfidemediated oligomerization by binding to specific chaperone proteins that are present in the IS. It is likely that these tetrameric core complexes can then traffic to the base of the OS, where they become incorporated into the newly forming disc membranes. At the base of the OS, disulfide-mediated oligomerization of these tetramers into intermediate complexes would then take place, presumably via the action of protein disulfide isomerase. Although our results are consistent with parts of this model, protein disulfide isomerase is known to be localized to the endoplasmic reticulum, but has not yet been reported in the OS. Our findings also confirm that both Rds and Rom-1 have protected and unpaired extracellular cysteines in the IS, which subsequently become accessible for inter-disulfide pairing in the OS. Such protection could be facilitated by association with chaperone proteins. Having unpaired cysteines appears to be a unique phenomenon to Rds and Rom-1, since all other members of the tetraspanin family are known to have fully paired cysteines from the time they form in the endoplasmic reticulum (37). In another study, we have directly addressed the role of intermolecular disulfide bond formation of Rds/Rom- 1 complexes known to be mediated by cysteine residue at the position 150 (Cys150) of Rds. We changed this cysteine to serine (C150S) in rods versus cones of transgenic mice and showed that C150S Rds cannot form higher-order oligomers or build the OS structures of rods or cones (our unpublished work). Therefore, we concluded that Rds oligomerization and outer segment morphogenesis are temporally linked and likely occur simultaneously.

The photoreceptor OS is undoubtedly one of the most complex organelles in the human body. Not only is the OS bombarded with photons of light on a relatively constant basis, but the OS also must undergo constant renewal $(38,39)$. In addition, though speculative at this point, it is tempting to propose that the OS renewal process may involve specific chaperones that help guide the trafficking of structural proteins needed for disc morphogenesis, as well as participating in the assembly and stabilization of higher order Rds/Rom-1 complexes formed in the OS. Future experiments to identify these putative chaperones will be of great importance in developing a more complete understanding of intracellular trafficking and membrane biogenesis in this highly polarized cell type. These postulated molecules also may be involved in processes that underlie the observed difference in Rds behavior in rods vs cones. It is possible that mutations in Rds hinder interactions with some of these chaperones, which in turn impairs higher-order complex formation and the development of varying disease phenotypes. This study has provided the first in vivo biochemical evidence documenting that intermediate and higher-order Rds oligomers are assembled in the OS and are not present in the IS.

\section{Supplementary Material}

Refer to Web version on PubMed Central for supplementary material. 


\section{Acknowledgments}

We are grateful to Barbara Nagel, Jeff Skaggs, and Alexander Quiambao for providing excellent technical assistance. We are also grateful to Dr. Robert Molday (University of British Columbia, Vancouver, BC) for providing antiGARP-4B1 monoclonal antibody. We also thank Drs. Shannon Conley and Heidi Stricker for their helpful comments on the manuscript.

\section{References}

1. Molday RS, Hicks D, Molday L. Peripherin. A rim-specific membrane protein of rod outer segment discs. Invest Ophthalmol Vis Sci 1987;28:50-61. [PubMed: 2433249]

2. Bascom RA, Manara S, Collins L, Molday RS, Kalnins VI, McInnes RR. Cloning of the cDNA for a novel photoreceptor membrane protein (rom-1) identifies a disk rim protein family implicated in human retinopathies. Neuron 1992;8:1171-1184. [PubMed: 1610568]

3. Sanyal S, Jansen HG. Absence of receptor outer segments in the retina of Rds mutant mice. Neurosci Lett 1981;21:23-26. [PubMed: 7207866]

4. Travis GH, Sutcliffe JG, Bok D. The retinal degeneration slow (Rds) gene product is a photoreceptor disc membrane-associated glycoprotein. Neuron 1991;6:61-70. [PubMed: 1986774]

5. Kajiwara K, Hahn LB, Mukai S, Travis GH, Berson EL, Dryja TP. Mutations in the human retinal degeneration slow gene in autosomal dominant retinitis pigmentosa. Nature 1991;354:480-483. [PubMed: 1684223]

6. Wells J, Wroblewski J, Keen J, Inglehearn C, Jubb C, Eckstein A, Jay M, Arden G, Bhattacharya S, Fitzke F, et al. Mutations in the human retinal degeneration slow (RDS) gene can cause either retinitis pigmentosa or macular dystrophy. Nat Genet 1993;3:213-218. [PubMed: 8485576]

7. Boesze-Battaglia K, Goldberg AF, Dispoto J, Katragadda M, Cesarone G, Albert AD. A soluble peripherin/Rds C-terminal polypeptide promotes membrane fusion and changes conformation upon membrane association. Exp Eye Res 2003;77:505-514. [PubMed: 12957149]

8. Goldberg AF, Molday RS. Defective subunit assembly underlies a digenic form of retinitis pigmentosa linked to mutations in peripherin/Rds and rom-1. Proc Natl Acad Sci USA 1996;93:13726-13730. [PubMed: 8943002]

9. Goldberg AF, Loewen CJ, Molday RS. Cysteine residues of photoreceptor peripherin/Rds: role in subunit assembly and autosomal dominant retinitis pigmentosa. Biochemistry 1998;37:680-685. [PubMed: 9425091]

10. Ding XQ, Nour M, Ritter LM, Goldberg AF, Fliesler SJ, Naash MI. The R172W mutation in peripherin/Rds causes a cone-rod dystrophy in transgenic mice. Hum Mol Genet 2004;13:2075-2087. [PubMed: 15254014]

11. Ding XQ, Stricker HM, Naash MI. Role of the second intradiscal loop of peripherin/Rds in homo and hetero associations. Biochemistry 2005;44:4897-4904. [PubMed: 15779916]

12. Poetsch A, Molday LL, Molday RS. The cGMP-gated channel and related glutamic acid-rich proteins interact with peripherin-2 at the rim region of rod photoreceptor disc membranes. J Biol Chem 2001;276:48009-48016. [PubMed: 11641407]

13. Farjo R, Skaggs JS, Nagel BA, Quiambao AB, Nash ZA, Fliesler SJ, Naash MI. Retention of function without normal disc morphogenesis occurs in cone but not rod photoreceptors. The Journal of cell biology 2006;173:59-68. [PubMed: 16585269]

14. Farjo R, Naash MI. The role of Rds in outer segment morphogenesis and human retinal disease. Ophthal Genet 2006;27:117-122.

15. Moritz OL, Molday RS. Molecular cloning, membrane topology, and localization of bovine rom-1 in rod and cone photoreceptor cells. Invest Ophthalmol Vis Sci 1996;37:352-362. [PubMed: 8603840]

16. Connell GJ, Molday RS. Molecular cloning, primary structure, and orientation of the vertebrate photoreceptor cell protein peripherin in the rod outer segment disk membrane. Biochemistry 1990;29:4691-4698. [PubMed: 2372552]

17. Goldberg AF, Moritz OL, Molday RS. Heterologous expression of photoreceptor peripherin/Rds and Rom-1 in COS-1 cells: assembly, interactions, and localization of multisubunit complexes. Biochemistry 1995;34:14213-14219. [PubMed: 7578020] 
18. Loewen CJ, Molday RS. Disulfide-mediated oligomerization of Peripherin/Rds and Rom-1 in photoreceptor disk membranes. Implications for photoreceptor outer segment morphogenesis and degeneration. J Biol Chem 2000;275:5370-5378. [PubMed: 10681511]

19. Clarke G, Goldberg AF, Vidgen D, Collins L, Ploder L, Schwarz L, Molday LL, Rossant J, Szel A, Molday RS, Birch DG, McInnes RR. Rom-1 is required for rod photoreceptor viability and the regulation of disk morphogenesis. Nat Genet 2000;25:67-73. [PubMed: 10802659]

20. Kedzierski W, Moghrabi WN, Allen AC, Jablonski-Stiemke MM, Azarian SM, Bok D, Travis GH. Three homologs of Rds/peripherin in Xenopus laevis photoreceptors that exhibit covalent and noncovalent interactions. J Cell Sci 1996;109(Pt 10):2551-2560. [PubMed: 8923216]

21. Weng J, Belecky-Adams T, Adler R, Travis GH. Identification of two Rds/peripherin homologs in the chick retina. Invest Ophthalmol Vis Sci 1998;39:440-443. [PubMed: 9478005]

22. Li C, Ding XQ, O’Brien J, Al-Ubaidi MR, Naash MI. Molecular characterization of the skate peripherin/Rds gene: relationship to its orthologues and paralogues. Invest Ophthalmol Vis Sci 2003;44:2433-2441. [PubMed: 12766040]

23. Loewen CJ, Moritz OL, Tam BM, Papermaster DS, Molday RS. The role of subunit assembly in peripherin-2 targeting to rod photoreceptor disk membranes and retinitis pigmentosa. Mol Biol Cell 2003;14:3400-3413. [PubMed: 12925772]

24. Nour M, Ding XQ, Stricker H, Fliesler SJ, Naash MI. Modulating expression of peripherin/Rds in transgenic mice: critical levels and the effect of overexpression. Invest Ophthalmol Vis Sci 2004;45:2514-2521. [PubMed: 15277471]

25. Humphries MM, Rancourt D, Farrar GJ, Kenna P, Hazel M, Bush RA, Sieving PA, Sheils DM, McNally N, Creighton P, Erven A, Boros A, Gulya K, Capecchi MR, Humphries P. Retinopathy induced in mice by targeted disruption of the rhodopsin gene. Nat Genet 1997;15:216-219. [PubMed: 9020854]

26. Mears AJ, Kondo M, Swain PK, Takada Y, Bush RA, Saunders TL, Sieving PA, Swaroop A. Nrl is required for rod photoreceptor development. Nat Genet 2001;29:447-452. [PubMed: 11694879]

27. Stricker HM, Ding XQ, Quiambao A, Fliesler SJ, Naash MI. The Cys $214 \rightarrow$ Ser mutation in peripherin/ Rds causes a loss-of-function phenotype in transgenic mice. Biochem J 2005;388:605-613. [PubMed: 15656787]

28. Farjo R, Fliesler SJ, Naash MI. Effect of Rds abundance on cone outer segment morphogenesis, photoreceptor gene expression, and outer limiting membrane integrity. J Comp Neurol 2007;504:619-630. [PubMed: 17722028]

29. Tan E, Wang Q, Quiambao AB, Xu X, Qtaishat NM, Peachey NS, Lem J, Fliesler SJ, Pepperberg DR, Naash MI, Al-Ubaidi MR. The relationship between opsin overexpression and photoreceptor degeneration. Invest Ophthal-mol Vis Sci 2001;42:589-600.

30. Goldberg AF, Molday RS. Subunit composition of the peripherin/Rds-rom-1 disk rim complex from rod photo-receptors: hydrodynamic evidence for a tetrameric quaternary structure. Biochemistry 1996;35:6144-6149. [PubMed: 8634257]

31. Goldberg AF, Molday RS. Expression and characterization of peripherin/Rds-rom-1 complexes and mutants implicated in retinal degenerative diseases. Methods Enzymol 2000;316:671-687. [PubMed: 10800708]

32. Rajala RV, McClellan ME, Ash JD, Anderson RE. In vivo regulation of phosphoinositide 3-kinase in retina through light-induced tyrosine phosphorylation of the insulin receptor beta-subunit. J Biol Chem 2002;277:43319-43326. [PubMed: 12213821]

33. Tam BM, Moritz OL, Papermaster DS. The $\mathrm{C}$ terminus of peripherin/Rds participates in rod outer segment targeting and alignment of disk incisures. Mol Biol Cell 2004;15:2027-2037. [PubMed: 14767063]

34. Damek-Poprawa M, Krouse J, Gretzula C, Boesze-Battaglia K. A novel tetraspanin fusion protein, peripherin-2, requires a region upstream of the fusion domain for activity. $\mathrm{J}$ Biol Chem 2005;280:9217-9224. [PubMed: 15591062]

35. Boesze-Battaglia K, Stefano FP, Fitzgerald C, Muller-Weeks S. ROM-1 potentiates photoreceptor specific membrane fusion processes. Exp Eye Res 2007;84:22-31. [PubMed: 17055485] 
36. Lee ES, Burnside B, Flannery JG. Characterization of Peripherin/Rds and Rom-1 Transport in Rod Photoreceptors of Transgenic and Knockout Animals. Invest Ophthalmol Vis Sci 2006;47:2150_ 2160. [PubMed: 16639027]

37. Hemler ME. Tetraspanin proteins mediate cellular penetration, invasion, and fusion events and define a novel type of membrane microdomain. Annu Rev Cell Dev Biol 2003;19:397-422. [PubMed: 14570575]

38. Goldberg AF. Role of peripherin/Rds in vertebrate photoreceptor architecture and inherited retinal degenerations. Int Rev Cytol 2006;253:131-175. [PubMed: 17098056]

39. Boesze-Battaglia K, Goldberg AF. Photoreceptor renewal: a role for peripherin/Rds. Int Rev Cytol 2002;217:183-225. [PubMed: 12019563] 

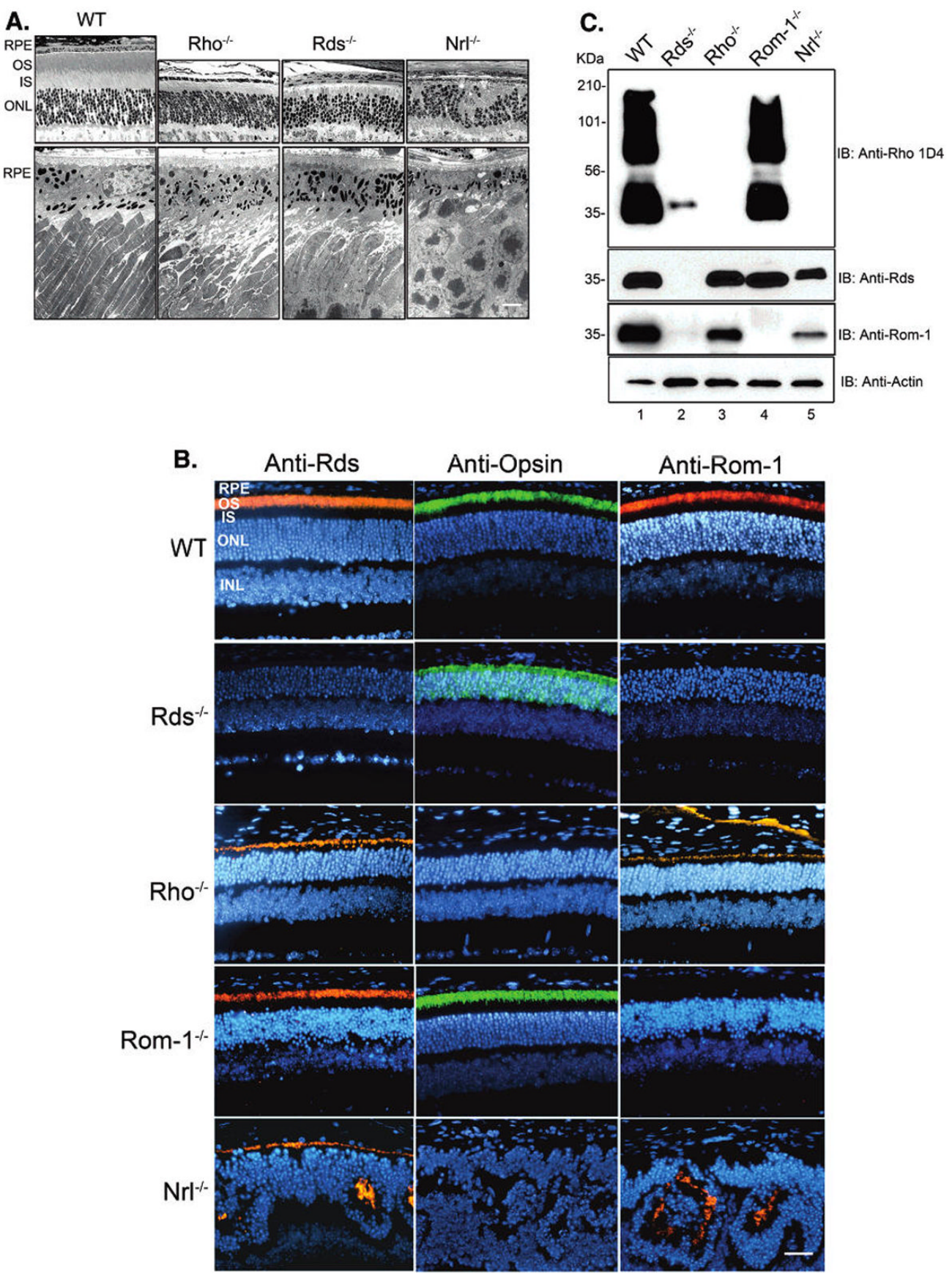

Figure 1.

Retinal morphology, expression levels and cellular localization of Rds and Rom-1 from mouse retinas. (A) Upper panels: LM images of retinas from 1-month old WT, $\mathrm{Rho}^{-/-} \mathrm{Rds}^{-/-}$, and $\mathrm{Nrl}^{-l-}$ mice. Lower panels: corresponding EM images of photoreceptor-RPE interface from these animals. Scale bar for LM and EM images are 25 and $4 \mu \mathrm{M}$, respectively. EM demonstrates lack of OSs in $\mathrm{Rho}^{-1-}$ and $\mathrm{Rds}^{-/-}$retinas. (B) Immunohistochemistry of retinas from 1-month old WT, $\mathrm{Rds}^{-/-}$, $\mathrm{Rho}^{-/-}$, $\mathrm{Rom}_{-1}{ }^{-/-}$, and $\mathrm{Nrl}^{-/-}$mice. Left-hand panels; anti-Rds immunoreactivity; middle panels: anti-rhodopsin; right-hand panels; anti-Rom-1. Nuclei were stained with DAPI (blue). Red and green fluorescence is due to Cy3 and FITC conjugated secondary antibodies. $\mathrm{Rho}^{-1-}$ retinas exhibit immunolabeling for Rds and Rom-1 at the tip of 
the connecting cilium. Abbreviations: RPE, retinal pigment epithelium; OS, outer segment; IS, inner segment; ONL, outer nuclear layer; INL, inner nuclear layer. (C) Western blot analysis of WT, $\mathrm{Rds}^{-1-}, \mathrm{Rho}^{-/-}, \mathrm{Rom}_{-} 1^{-/-}$, and $\mathrm{Nrl}^{-1-}$ mouse retinas at $\mathrm{p} 30$, probed with antibodies against rhodopsin, Rds, Rom-1, and actin (control). Note marked reduction of anti-rhodopsin immunostaining in the $\mathrm{Rds}^{-1-}$ retina (photoreceptor dysplasia, absence of OSs) and coordinate loss of Rom-1 expression. In $\mathrm{Nrl}^{-/-}$retina, note lack of rhodopsin expression (lacks rod photoreceptors) and also reduced Rom-1/Rds ratio compared to WT and $\mathrm{Rho}^{-1-}$ retinas. Numerical values in the left-hand margin $\left(M_{\mathrm{r}}\right.$, in $\left.\mathrm{kDa}\right)$ of each panel indicate the migration positions of protein molecular weight markers. 

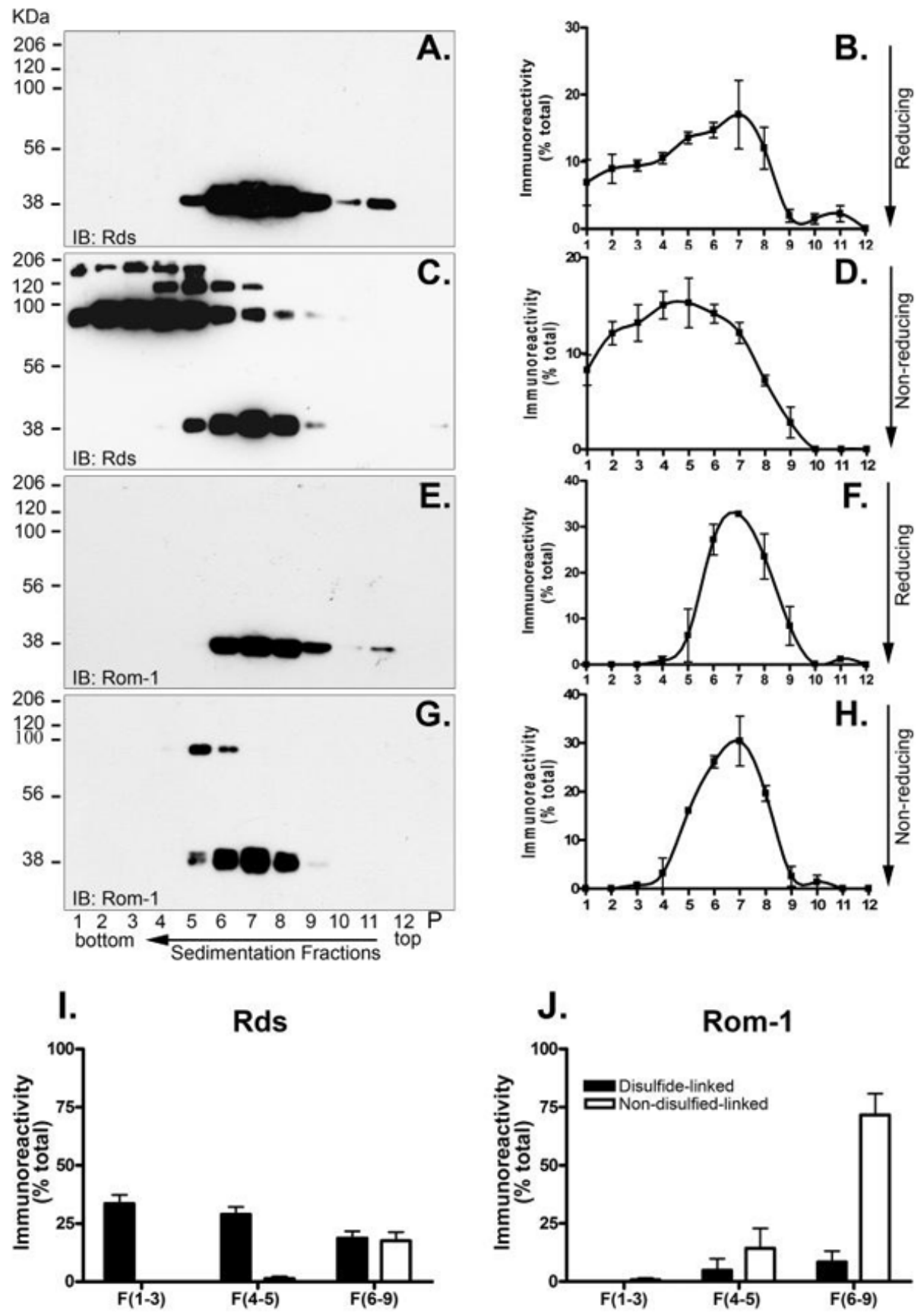

Figure 2.

Subunit assembly of Rds and Rom-1 in the rod-dominant WT retina. Whole WT retinas (200 $\mu \mathrm{g}$ of protein) were solubilized in the presence of either DTT (reducing conditions, panels A and E) or NEM (nonreducing conditions, panels $\mathrm{C}$ and $\mathrm{G}$ ). Extracts were sedimented under reducing (panels $\mathrm{A}$ and $\mathrm{E}$ ) or nonreducing (panels $\mathrm{C}$ and $\mathrm{G}$ ) conditions. Western blot analysis was performed on all fractions and probed with anti-Rds (panels $\mathrm{A}$ and $\mathrm{C}$ and corresponding quantification in panels B and D) and anti-Rom-1 (panels E and G and corresponding quantification in panels $\mathrm{F}$ and H). Levels of Rds (panel I) and Rom-1 (panel J) present in noncovalently linked vs disulfide-linked complexes were averaged from three independent experiments (results presented as \% of the total). Under reducing conditions, a single peak with 
tetrameric stoichiometry was observed for both proteins, spanning fractions 5 to 10 . Under nonreducing conditions, higher-order Rds homo-oligomers linked through disulfide bonds were detected in fractions 1-4. By contrast, Rom-1 mainly appeared as one main peak corresponding to tetrameric complexes, with only a small portion representing disulfide-linked complexes detected in fractions 5 and 6 . Numerical values in the left-hand $\operatorname{margin}\left(M_{\mathrm{r}}\right.$, in $\left.\mathrm{kDa}\right)$ of each panel indicate the migration positions of protein molecular weight markers. 
A.

C.
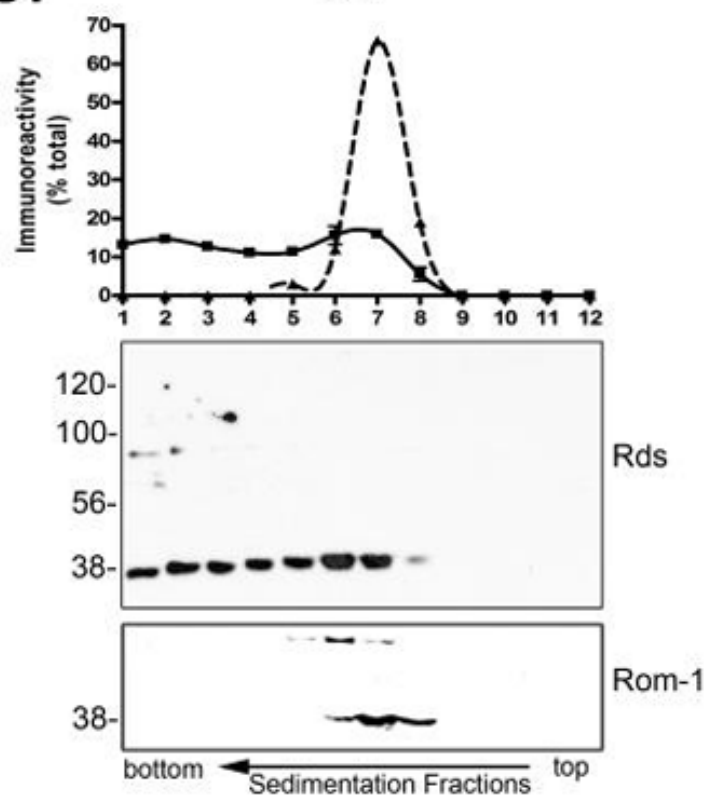

Figure 3.
B.
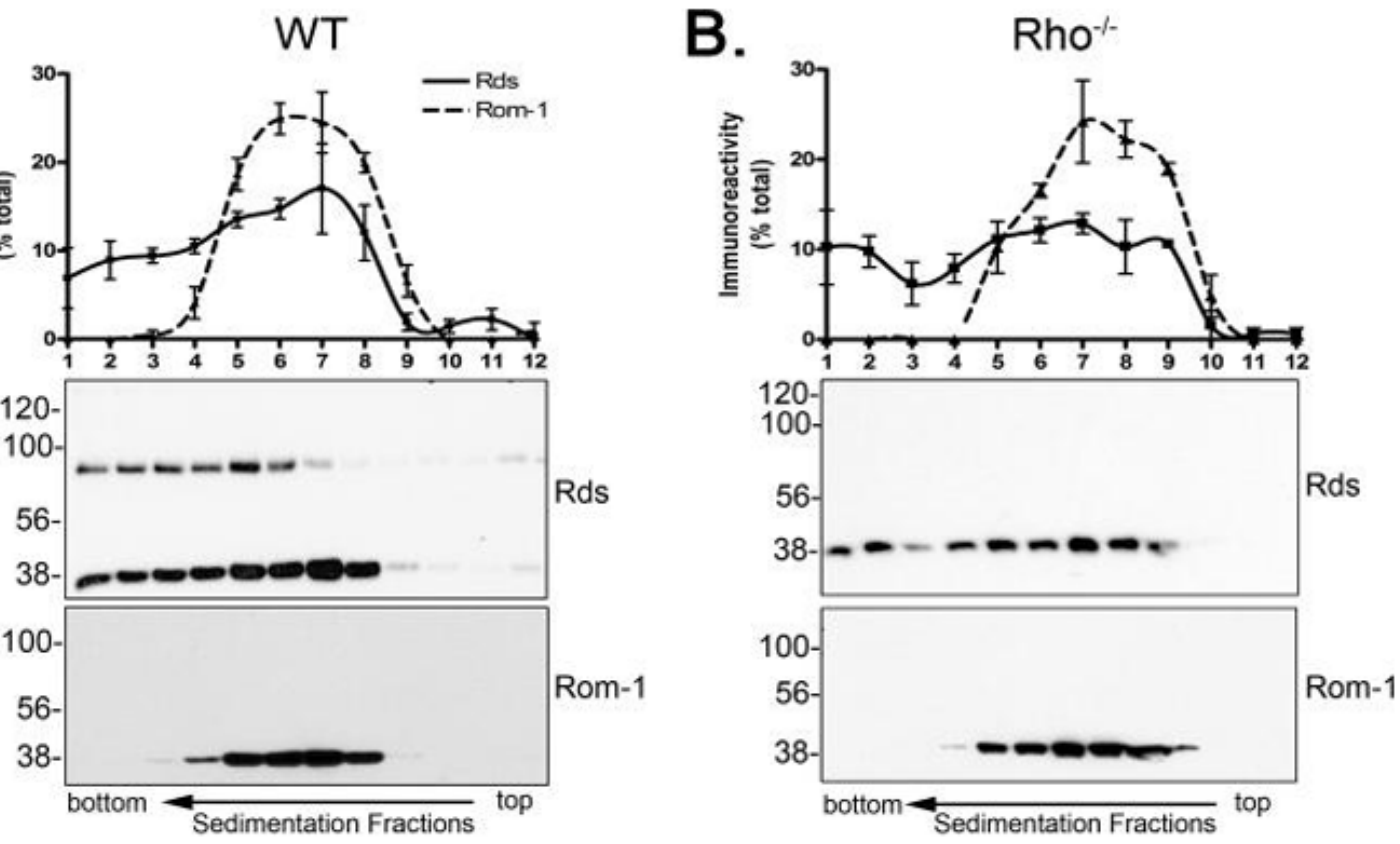

D.

Rom-1/-

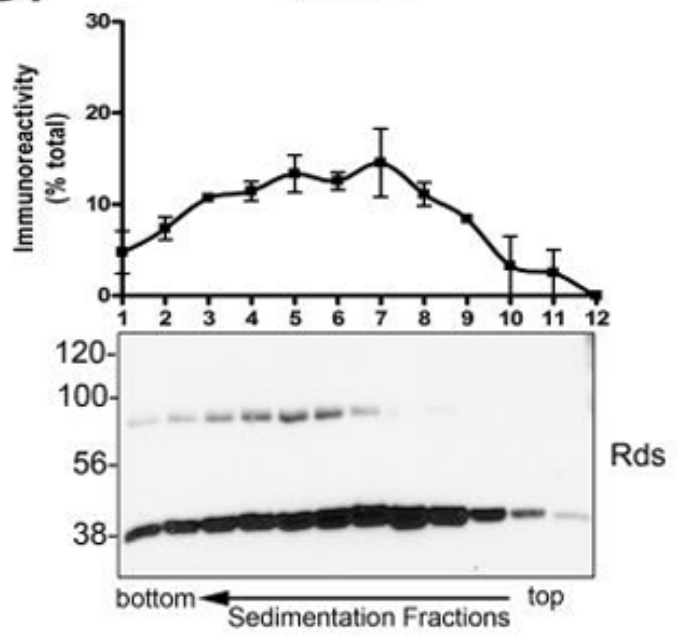

Subunit assembly of Rds and Rom- 1 in retinas from knockout mice. Whole retina extracts (200 $\mu \mathrm{g}$ of protein each) from WT (A), $\mathrm{Rho}^{-/-}$(B), $\mathrm{Nrl}^{-/-}$(C), and $\mathrm{Rom}^{-1^{--}}$(D) mice were sedimented under nonreducing conditions. Identical Western blots under reducing conditions of the fractions from each extract were probed with anti-Rds and anti-Rom-1 antibodies. Rds homo-oligomeric complexes are much less abundant in the $\mathrm{Rho}^{-1-}$ retina compared to WT, and the majority of Rds and Rom- 1 forms homo- and hetero-tetrameric complexes. In contrast, $\mathrm{Nrl}^{-/-}$and Rom- $1^{-/-}$retinas show WT-like sedimentation patterns with respect to Rds distribution and oligomerization. Numerical values in the left-hand margin $\left(M_{\mathrm{r}}\right.$, in $\left.\mathrm{kDa}\right)$ of each panel indicate the migration positions of protein molecular weight markers. 

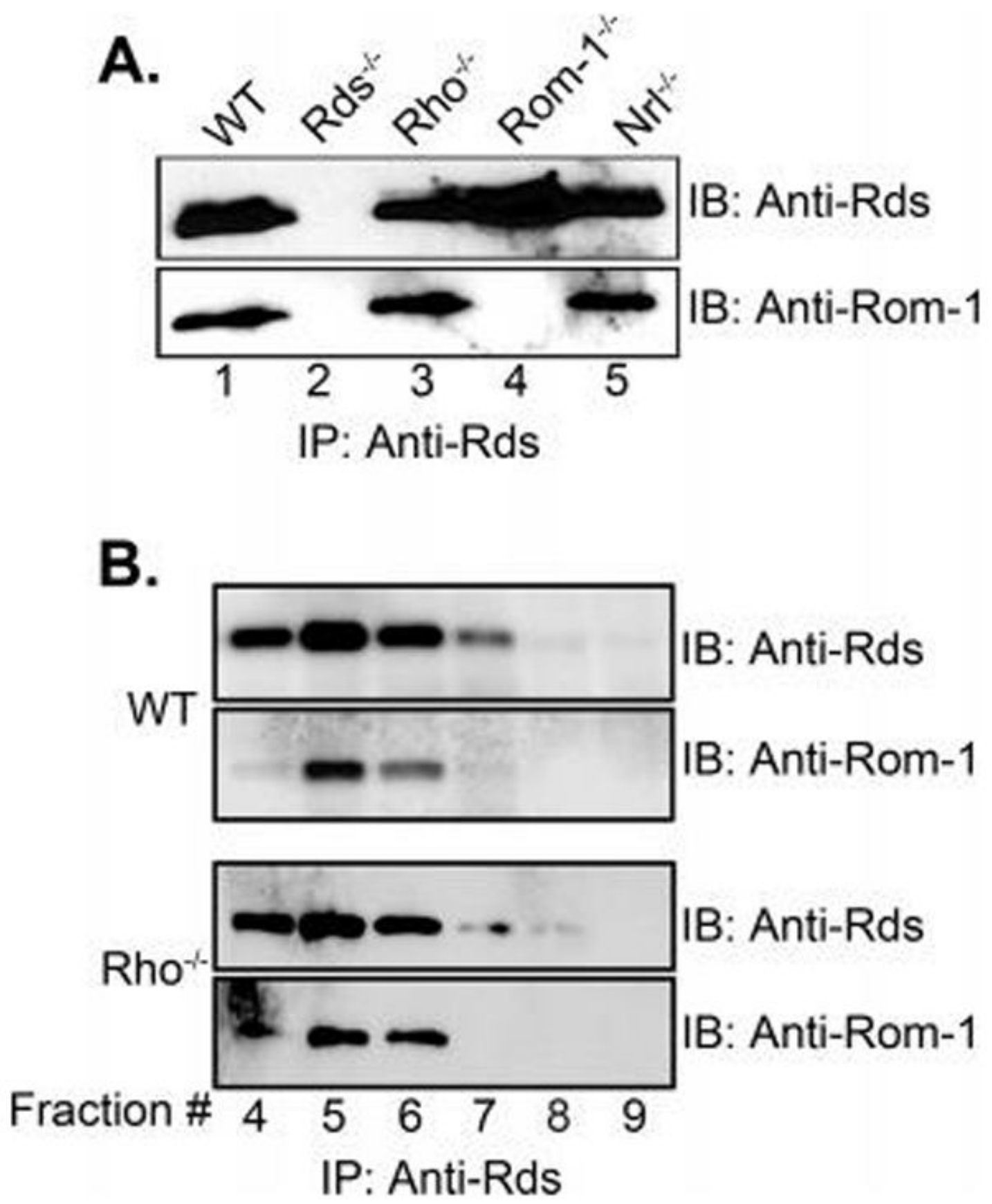

Figure 4.

Association of Rds and Rom-1 in retinas from different mouse strains. (A) Immunoprecipitation of retinal extracts from all models used in this study with anti-Rds, and corresponding Western blots probed with anti-Rds (upper panel) and anti-Rom-1 (lower panel) antibodies. Rds and Rom-1 interact in IS-enriched $\mathrm{Rho}^{-/-}$(OS-free) and $\mathrm{Nrl}^{-/-}$(pure cone, rod-free) retinas. Lanes are numbered 1-5 to reflect the genotype of the extract loaded in each lane. (B) Western blot analysis of immunoprecipitates of fractions 4-9 from WT and Rho ${ }^{-/}$ retinas. Blots were probed with anti-Rds and anti-Rom-1. Fractions 5 and 6 represent mostly Rds/Rom-1 hetero-tetramers. Lanes are numbered 4-9 to reflect the fraction number obtained from the sucrose gradient sedimentation. 

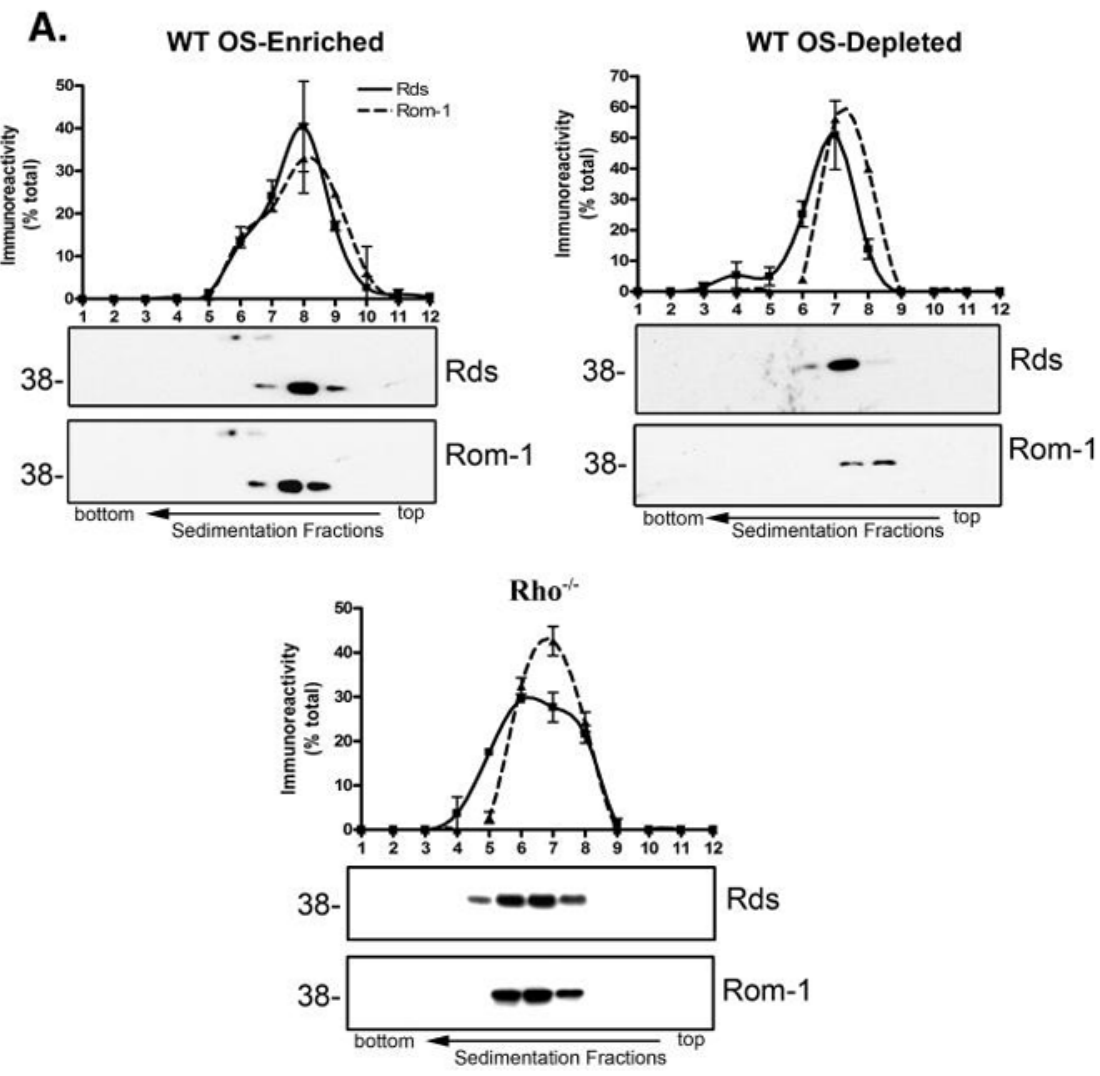

B.
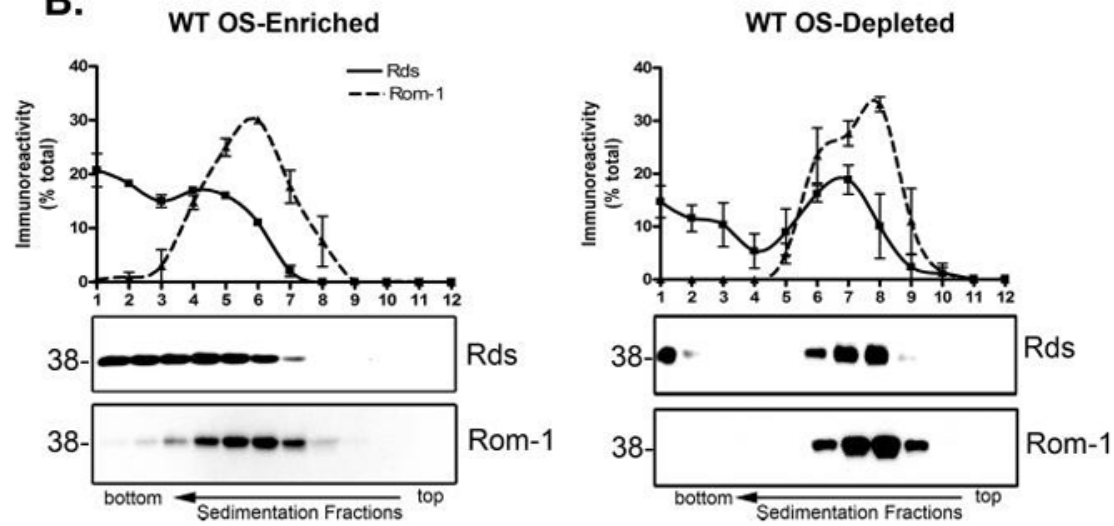

Figure 5.

Oligomerization of Rds in the outer segment. (A) Reducing velocity sedimentation analysis of OS-enriched and OS-depleted preparations from WT retinas and retinal extracts from $\mathrm{Rho}^{-1-}$ mice. Immunoblots were probed with anti-Rds and anti-Rom- 1 antibodies. In the presence of DTT, higher-order complexes of Rds and Rom-1 were reduced to tetramers that appeared as a single peak. (B) Nonreducing velocity sedimentation of OS-enriched and OS-depleted preparations from WT retinas. Note mainly the presence of higher-order complexes of Rds in the OS-enriched preparation, whereas the OS-depleted samples show mainly the presence of tetrameric forms. The migration position of Rds and Rom-1 monomers $\left(M_{\mathrm{r}} \sim 38 \mathrm{kDa}\right)$ is indicated. 

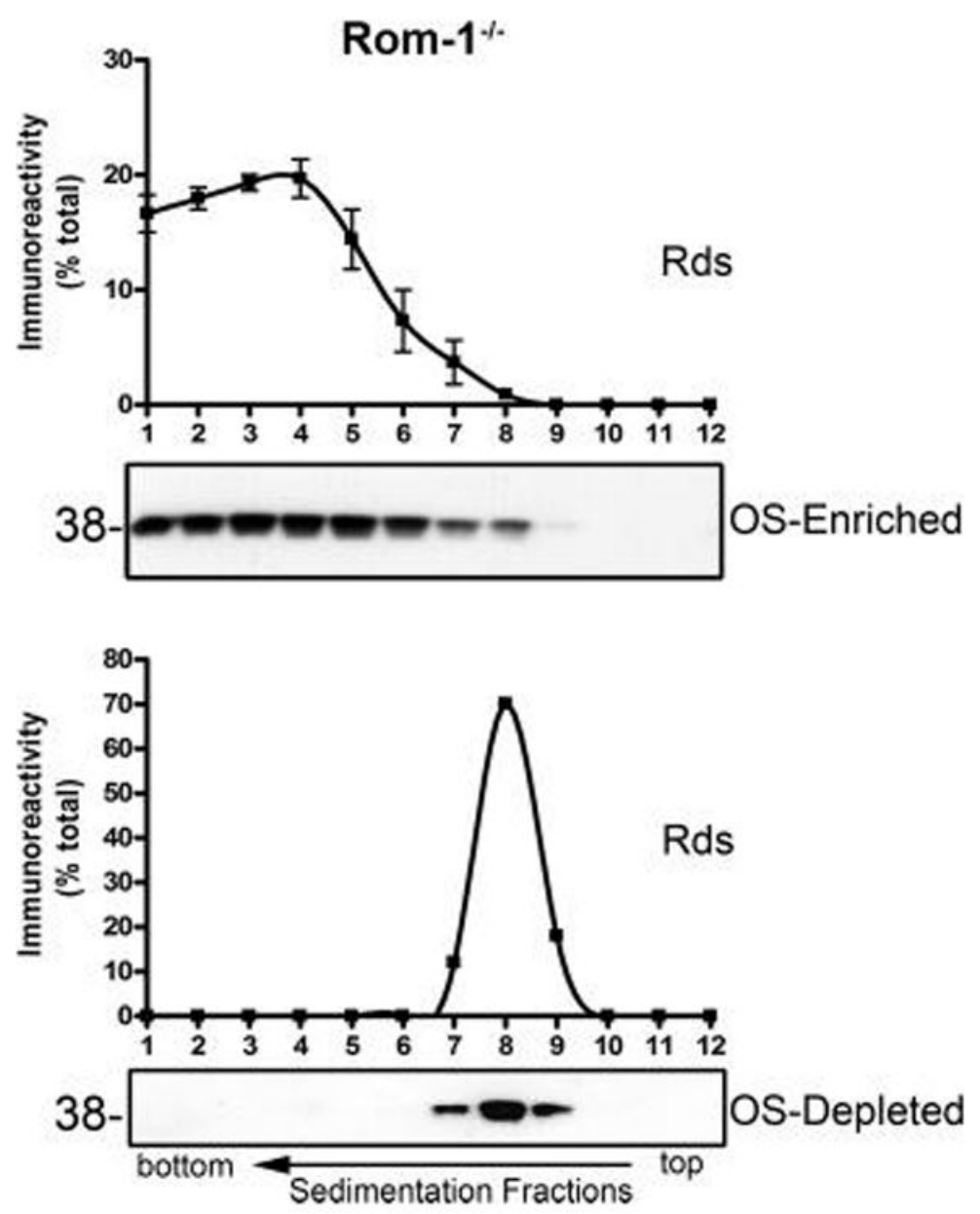

Figure 6.

Rds complex assembly in the absence of Rom-1. Velocity sedimentation analysis of OSenriched and OS-depleted fractions from Rom- $1^{-/-}$retinas performed under nonreducing conditions and immunobloted with anti-Rds antibody. The majority of Rds is present in octamers and higher-order homo-oligomers (fractions 1-6), similar to findings with WT OS preparations. Note the complete lack of Rds higher-order oligomers in the OS-depleted samples from Rom- $1^{-/-}$retina, which contain mainly Rds homo-tetramers (fraction 8). This finding suggests that Rds tetrameric form is the preferred complex that traffics Rds from the IS to the OS, Rds homo-oligomers are essential for OS rim structure formation, and that Rds higherorder oligomerization occurs in the OS. The migration position of Rds $\left(M_{\mathrm{r}} \sim 38 \mathrm{kDa}\right)$ is indicated. 
A.

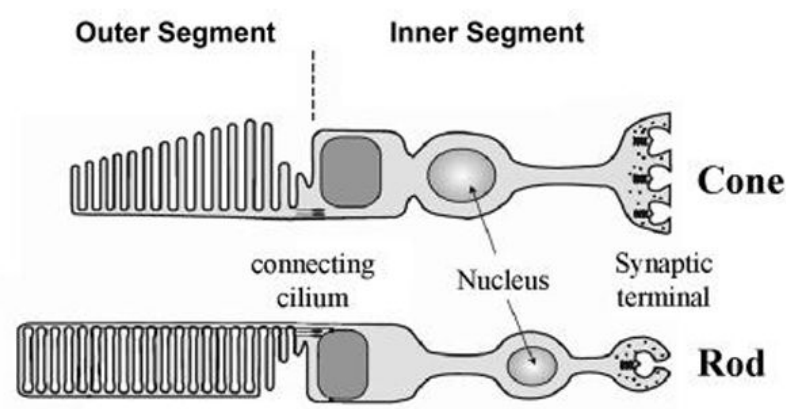

B.

OS-Enriched

OS-Depleted
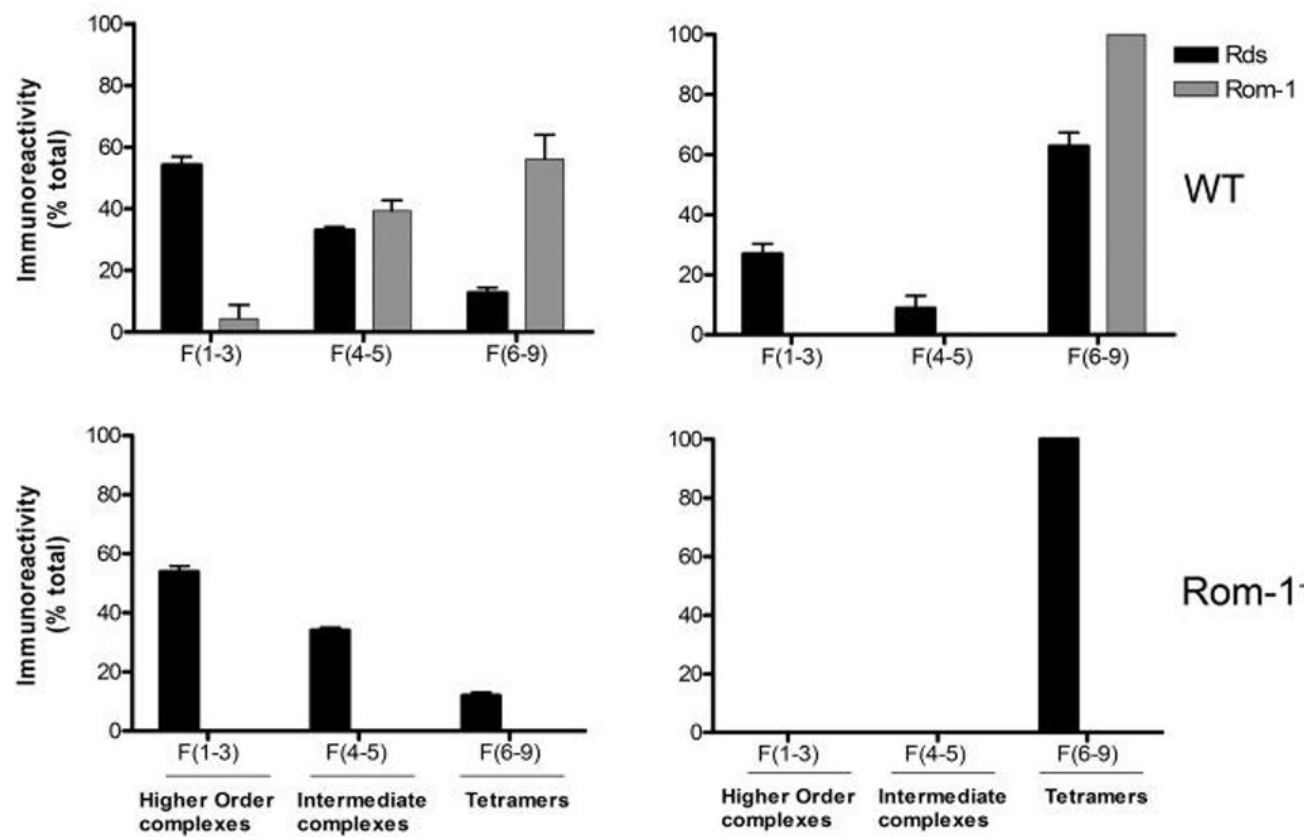

Rom-1 ${ }^{-1}$

Figure 7.

Summary illustration depicting the sites of Rds and Rom-1 complex formation in rod and cone photoreceptor cells. (A) Structural diagram of rod and cone photoreceptor cell. (B) Distribution of Rds complexes in OS-enriched and OS-depleted preparations from WT and Rom- $1^{-1-}$ retinas. The histograms summarize the results of the present study. Depending on the nature of the complex, Rds was recovered mostly as covalent disulfide-linked high order oligomers in the OS and noncovalent tetrameric complex in the IS from both models. Some of these complexes are homo complexes of Rds or hetero associations with Rom-1. The rearrangement from a noncovalent tetrameric complexes formed in the IS to a covalent octameric and oligomeric complexes formed in the OS would require the enzymatic action of an as yet unknown disulfide isomerase that should be located in the OS. 